\title{
Fluctuating Asymmetry in Root Number and Morphology of Permanent Premolars and Molars-Case Reports
}

\author{
I. Anand Sherwood ${ }^{1}$ and P. Govinda Reddy ${ }^{2}$ \\ ${ }^{1}$ Department of Conservative Dentistry and Endodontics, CSI College of Dental Sciences, Madurai - 02, Tamil \\ Nadu, India \\ ${ }^{2}$ Department of Anthropology, University of Madras, Chennai, India
}

\begin{abstract}
Aim of this article is to report cases with fluctuating asymmetry in root morphology and root number in permanent premolars and molars and to survey the literature about reporting for fluctuating asymmetry in roots of teeth. Fluctuating asymmetry is a left-right asymmetry of a paired structure that is usually symmetrical. Teeth in corresponding quadrants of the upper and lower jaws are normally symmetrical structures that exhibit mirror imagery. Fluctuating asymmetry does occur at varying levels for all root traits. Fluctuating asymmetry for root morphology and number has been poorly studied. In this article, 21
\end{abstract}

Van Valen (1962) grouped deviations from perfect symmetry in an organism into three categories: (1) directional asymmetry; (2) antisymmetry; and (3) fluctuating asymmetry (an asymmetry involving a paired structure that is usually symmetrical). Fluctuating asymmetry may be either quantitative or qualitative (Lundström 1961). Bilateral asymmetry in root number is quantitative variation and root morphology asymmetry is qualitative variation.

Fluctuating asymmetry has been associated with congenital abnormalities, genetic syndromes, and elevated levels of inbreeding, and it is also thought to be a general indicator of stress caused by nutritional and/ or disease during development (Scott and Turner 2000). Teeth in corresponding quadrants of the upper and lower jaws are normally symmetrical structures that exhibit mirror imagery. Asymmetry does occur, at varying levels, for all crown and root traits, including overall tooth size, cuspal dimensions, hypodontia and hyperdontia. Numerous studies have explored both the quantitative and qualitative nature of fluctuating asymmetry in crowns of the teeth (both size and morphological variation) among various populations (Lundström 1963; Garn et al. 1966; Dibennardo and Bailit 1978; Baume 1979; Townsend 1981; Noss et al. 1983; Kieser et al. 1986; Kieser and Groeneveld 1988; Scott and Turner 2000; Wetherell et al. 2004). But, with regard to fluctuating asymmetry for root number and morphology, studies are very limited (Sabala et al. 1994). cases with fluctuating asymmetry in root number and morphology of permanent premolars and molars are reported. Key points are: (1) Fluctuating asymmetry seems to be occurring in Tamil speaking population of Madurai South India more frequently than reported for Caucasian populations. (2) More detailed study of variation in root morphology with greater emphasis on fluctuating asymmetry for root morphology is warranted. 3) Fluctuating asymmetry is of importance to clinical dentists, dental morphologists, and dental anthropologists. Dental Anthropology 2011;24(1):16-24.

The present cases highlight the importance of the clinician and researcher to understand this phenotypic variation. A total of 21 cases are described exhibiting macroscopic fluctuating asymmetry in root number and morphology of permanent premolars and molars. This report describes four mandibular second premolars with extra roots, seven mandibular first molars with extra roots, four mandibular second molars with C-shaped roots, two mandibular second molars with extra roots, three mandibular third molars with extra roots, and one maxillary second molar with extra root configurations.

The subjects reported here were treated in the Department of Conservative Dentistry and Endodontics in the CSI College of Dental Sciences, Madurai, India, or a private referral clinic in Madurai, India, during the period between year June 2007 and December 2010 and were from the local indigenous Tamil speaking population. The bilateral eccentric periapical radiographs of patients who visited for treatment of either pain or caries were obtained (30-degree mesial angulation using a protractor). All the periapical radiographs were taken using an X-Mind ${ }^{\circledR}$ AC X-ray generator (Satelec Acteon Group, Gustave Eiffel, France) operated at $70 \mathrm{kVp}$ and 8

Correspondence to: I. Anand Sherwood, "Anand Dental Clinic", Number 1, Meenakshi Towers, P. T. Rajan Road, Bibikulam, Madurai - 02, Tamil Nadu, India

E-mail: anand.sherwood@gmail.com 


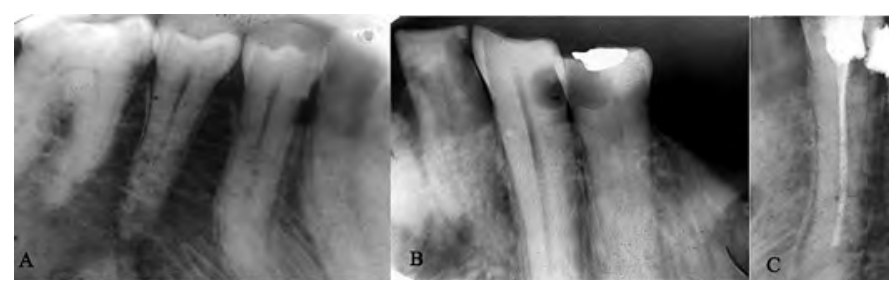

Fig. 1. (Left) Mandibular left second premolar. (Center) Mandibular right second premolar of the same case (diagnostic radiograph). (Right) Postoperative mandibular right second premolar showing two roots. Case is a 45 year old female.

mA. Size 2 periapical Kodak dental films (Eastman Kodak Co., Rochester, NY) were used. Periapical radiographs were taken using paralleling cone technique and films were held using film holders. Panoramic radiographs used in this report were taken using Planmeca x-ray unit (Proline EC, Helsinki, Finland). Films were developed using manual X-ray developer and fixer (Eastman Kodak Co., Rochester, NY) by the time and temperature method. The radiographs were placed on a viewing box, and the light surrounding the radiograph was blocked. Each radiograph was independently studied by two independent reviewers by using magnifying lens (3X). Disagreement in the interpretation of images was discussed with two endodontists, and a consensus was reached (Tu et al. 2007, Schafer et al. 2009). The criteria for the indication of an extra root were justified by crossing the translucent lines, defining the pulp space and the periodontal ligaments (Walker and Quackenbush 1985).

\section{Mandibular Second Premolars}

\section{Report 1}

A 45 year old female reported to the department with the complaint of pain in the mandibular right first and second premolars, a diagnosis of irreversible pulpitis was made. On examination, the periapical radiograph (Fig. 1B) of the mandibular right second premolar showed that the tooth had two separate roots in mesiodistal orientation.

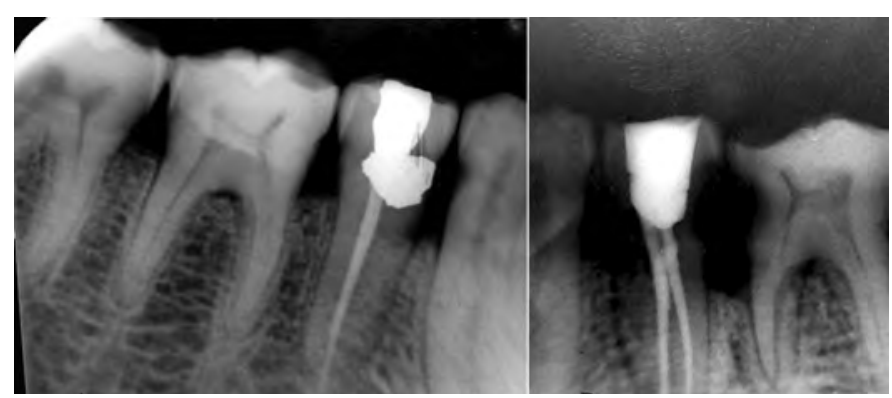

Fig. 2. (Left) Mandibular left second premolar. (Right) Postoperative mandibular right second premolar showing two roots. Case is a 35 year od male.

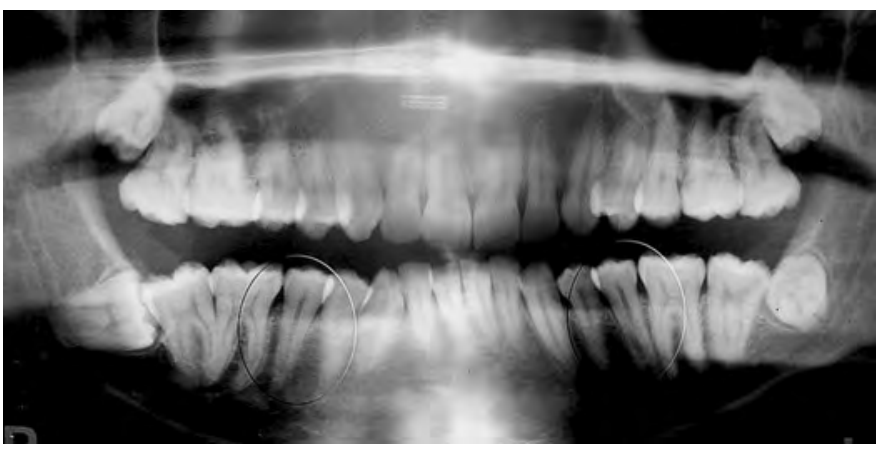

Fig. 3. Panoramic radiograph showing mandibular left second premolar with two roots and mandibular right second premolar with only one root. Case is a 21 year old female.

This was confirmed during root canal treatment (Fig. 1C). A contralateral radiograph (Fig. 1A) was taken for the mandibular left second premolar, and it had only one root.

\section{Report 2}

A 35 year old male was referred with the complaint of pain in both right and left mandibular second premolars. A diagnosis of irreversible pulpitis due to carious pulp exposure was made. On examination of radiographs (Fig. 2B) the mandibular right second premolar had two separate roots in mesiodistal orientation while the mandibular left second premolar (Fig. 2A) had only one root.

\section{Report 3}

A 21 year old female reported with a complaint of pain in the mandibular right third molar region, and a panoramic radiograph was advised. On examination, pain was diagnosed as being due to a mandibular right third molar impaction. It was noted on the panoramic radiograph (Fig. 3) that the mandibular left second premolar had two separate roots in mesiodistal orientation, whereas the mandibular right second premolar had only one root.

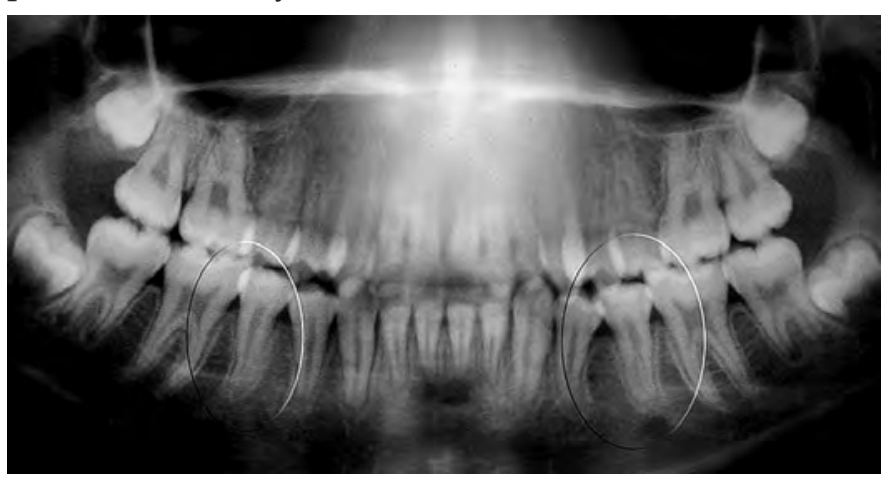

Fig. 4. Panoramic radiograph showing mandibular left second premolar with two roots and mandibular right second premolar with only one root. Case is a 16 year old female. 

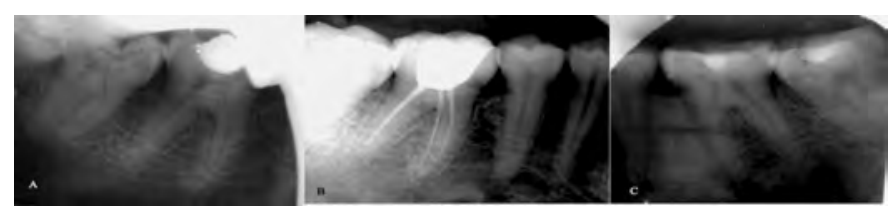

Fig. 5. (Left) Preoperative diagnostic radiograph showing presence of radix paramolaris on mandibular left first molar. (Center) Post-obturation radiograph confirming the presence of a radix paramolaris on mandibular left first molar. (Right) Radiograph of mandibular right first molar shows no presence of an extra root. Case is a 34 year old male.

\section{Report 4}

A 16 year old female was referred by an orthodontist for restoration and management of dental caries in the mandibular right and left first molars. It was noted that the patient had dentinal occlusal surface caries on the mandibular right and left second first molars, and they were managed with class I composite restorations. On examination of a panoramic radiograph (Fig. 4) taken for orthodontic purposes, it was noted that the mandibular left second premolar had two separate roots in mesiodistal orientation, whereas the mandibular right second premolar had only one root.

All the mandibular second premolars with extra root configuration in this report had Vertucci Type IV canal configuration (Vertucci 1984).

\section{Mandibular First Molars}

\section{Report 5}

A 34 year old male reported with a complaint of pain in the mandibular left first molar. It was noted on the radiograph that a class I amalgam restoration was impinging on the mesial pulp horn; a diagnosis of irreversible pulpititis was made and root canal treatment was undertaken. The periapical radiograph (Fig. 5A) showed that the mandibular left first molar had an extra root, a radix paramolaris (Carlsen and Alexandersen 1991). The contralateral periapical radiograph (Fig. 5C)

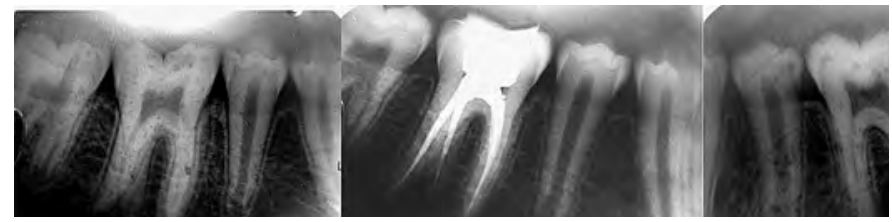

Fig. 6. (Left) Preoperative diagnostic radiograph of a mandibular right first molar showing the presence of a radix entomolaris. (Center) Post-obturation radiograph confirming the presence of a radix entomolaris on the mandibular right first molar. (Right) Radiograph of the mandibular left first molar showing the absence of anyextra root. Case is a 15 year old male.

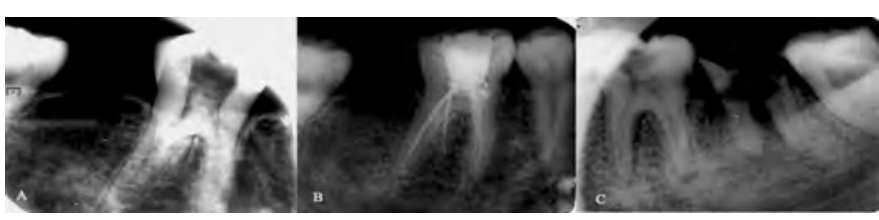

Fig. 7. (Left) Preoperative diagnostic radiograph showing the presence of a radix entomolaris on the mandibular left first molar. (Center) Post-obturation radiograph confirming the presence of a radix entomolaris on the mandibular left first molar. (Right) Radiograph of the mandibular right first molar showing no extra root. Case is a 27 year old female.

showed that that tooth had only two roots. Presence of a radix paramolaris was confirmed during root canal treatment of the mandibular left first molar (Fig. 5B).

\section{Report 6}

A 15 year old male was referred with pain in the mandibular right first molar and was diagnosed with irreversible pulpitis and root canal treatment was undertaken. The periapical radiograph of the mandibular right first molar (Fig. 6A) showed that the tooth had an extra root, a radix entomolaris (Carlsen and Alexandersen 1990). But the contralateral tooth (Fig. 6C) had only two roots. Presence of a radix entomolaris in mandibular right first molar was confirmed during the root canal treatment (Fig. 6B).

\section{Report 7}

A 27 year old female reported to the department with the chief compliant of pain in the mandibular left first molar. She was diagnosed with irreversible pulpitis and root canal treatment was undertaken. The periapical radiograph (Fig. 7A) disclosed that the mandibular left first molar had an extra root, a radix entomolaris. The contralateral periapical radiograph (Fig. 7C) showed that that tooth had only two roots. Presence of a radix entomolaris on the mandibular left first molar was confirmed during root canal treatment (Fig. 7B).

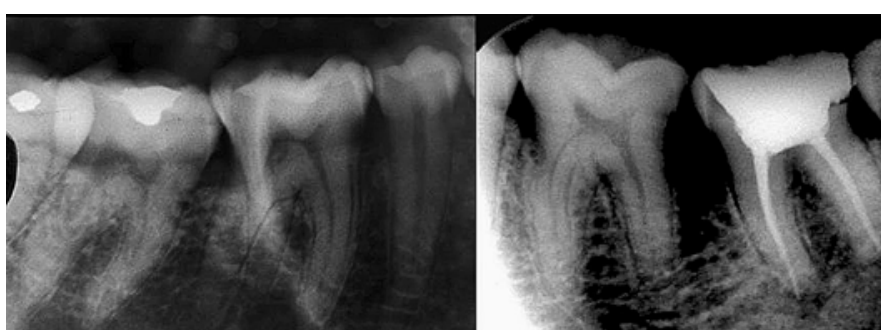

Fig. 8. (Left) Radiograph showing the presence of a radix entomolaris on the mandibular right first molar. (Right) Radiograph of the mandibular left first molar showing no extra root. Case is a 36 year old male. 


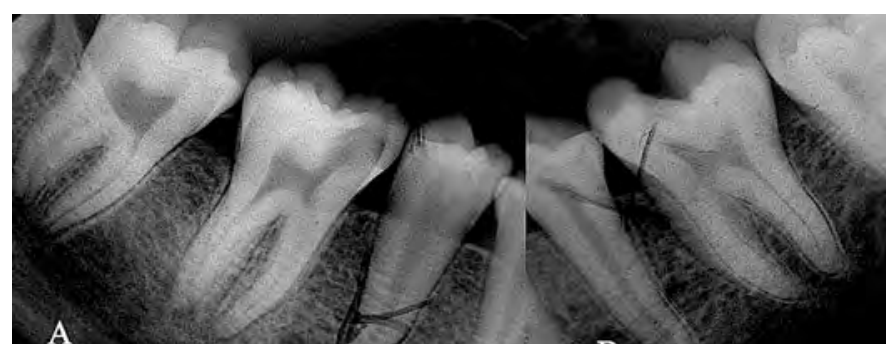

Fig. 9. Radiograph showing the presence of a radix entomolaris on the mandibular right first molar, but not on the mandibular left first molar. Case is a 19 year old male.

\section{Report 8}

A 36 year old male reported for full crown restoration of the mandibular right second molar, and he also had sensitivity in the mandibular left second molar. A diagnostic periapical radiograph was taken for both mandibular right and left second molar. It was concluded that root canal treatment in the mandibular right second molar was indicated, and a full crown restoration was undertaken. For the mandibular left second molar, sensitivity was due to cervical abrasion and a conservative treatment of desensitizing toothpaste was prescribed followed by evaluation at three months. From the periapical radiograph (Fig. 8A), it was noted that the mandibular right first molar had an extra root , a radix entomolaris, whereas the contralateral tooth (Fig. 8B) had only two roots.

\section{Report 9}

A 19 year old male was referred for root canal treatment of the mandibular right first molar. On clinical and radiographic examination a diagnosis of irreversible pulpitis was made and the subject was advised to have root canal treatment. It was noted on the periapical radiograph that the mandibular right first molar had an extra root radix entomolaris (Fig. 9A). On the contralateral tooth (Fig. 9.B) there were only two roots.

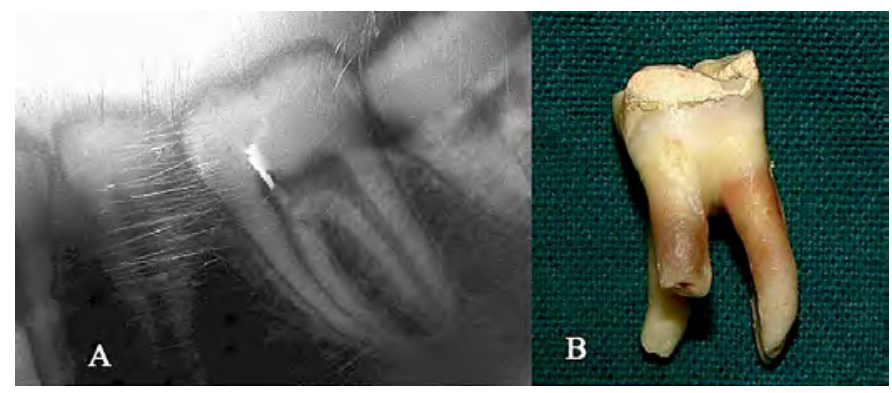

Fig. 10. (Left) Periapical radiograph of a mandibular left first molar showing no extra root. (Right) Extracted mandibular right first molar having an extra root radix entomolaris. Case is a 32 year old male.

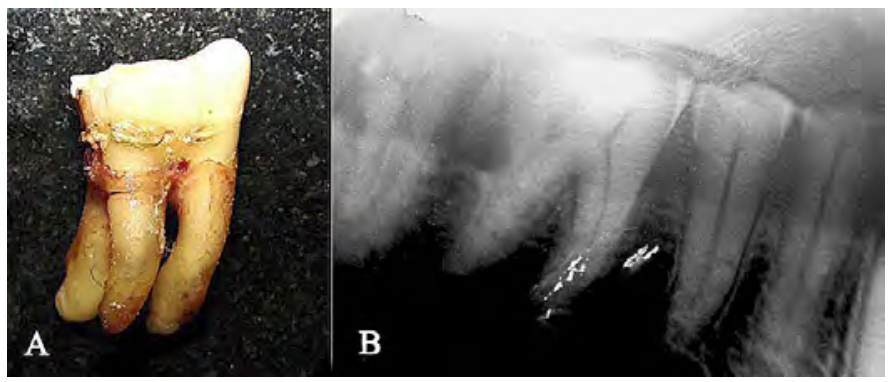

Fig. 11. (Left) An extracted mandibular left first molar having an extra root radix entomolaris. (Right) Radiograph of mandibular right first molar showing no extra root radix entomolaris. Case is a 50 year old male.

\section{Report 10}

A 32 year old male was referred with pain and deep caries on the mandibular right first molar; he was advised for root canal treatment. He rejected this, and the tooth was extracted. It was noted after extraction that the tooth had an extra root, a radix entomolaris (Fig. 10B). The contralateral periapical radiograph showed that that tooth had only two roots (Fig. 10.A).

\section{Report 11}

A 50 year old male was referred for root canal treatment of the mandibular left first molar, but the man wanted the tooth extracted. After extraction, it was noted that the tooth had an extra root, a radix entomolaris (Fig. 11A). The contralateral periapical radiograph was taken, and it showed that there were only two roots (Fig. 11B).

Based on Ribeiro and Consolaro's (1997) classification of radix entomolaris curvature, cases 5, 6, 7, 8, 9 had Type I curve, case 10 and 11 had Type II curve.

\section{Mandibular Second Molars \\ C-shaped root configuration}

\section{Report 12}

A 22 year old female with a complaint of pain in the mandibular left first molar was diagnosed with irreversible pulpitis, and root canal treatment was undertaken. The periapical radiograph disclosed that the

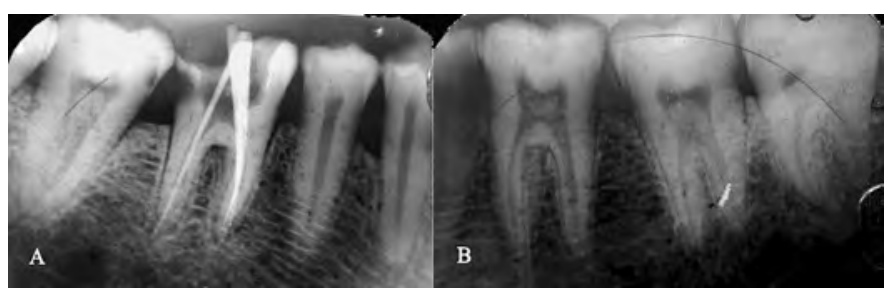

Fig. 12. (Left) Periapical radiograph showing the presence of C-shaped root on the mandibular left second molar. (Right) Radiograph of the mandibular right second molar showing two separate roots. Case is 22 year old female. 


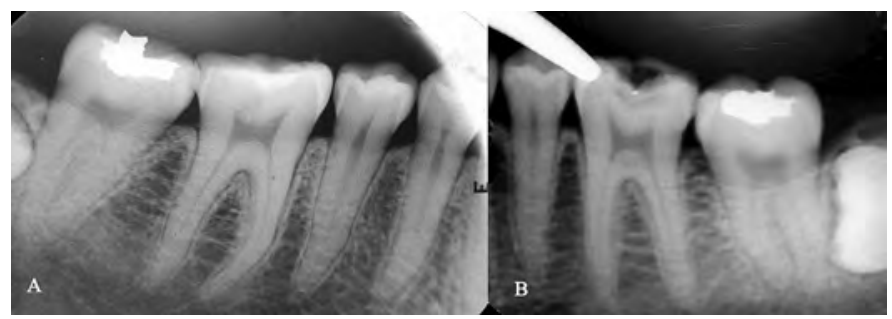

Fig. 13. (Left) Radiograph showing the presence of a C-shaped root on the mandibular right second molar. (Right) Radiograph of the mandibular left second molar showing two separate roots. Case is 43 year old male.

mandibular left second molar (Fig. 12A) had a C-shaped root configuration. According to the classification of Fan et al. (2004), it was a Type I. The contralateral tooth (Fig. 12B) had two separate roots.

\section{Report 13}

A 43 year old male reported to the department with a complaint of postoperative pain after a class I amalgam restoration on the mandibular right second molar. A periapical radiograph was taken, and it was concluded that pain was due to occlusal interference which was relieved. It was noted on the periapical radiograph that this tooth (Fig. 13A) had a C-shaped root configuration. According to Fan et al. (2004), the root was Type I. A contralateral radiograph (Fig. 13B.) was taken, and that tooth had two separate roots.

\section{Report 14}

A 34 year old female with a complaint of pain in the mandibular right first molar was referred for root canal treatment. This tooth had two separate roots (Fig. 14B). The contralateral tooth had a C-shaped root configuration (Fig. 14A). According to Fan et al. (2004), the root was of Type I.

\section{Report 15}

A 37 year old male reported with a complaint of pain in the mandibular left second molar. He was advised for root canal treatment, but he was not willing, and elected

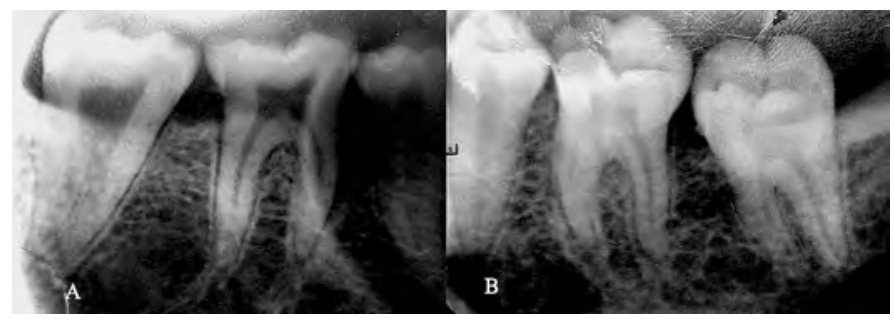

Fig. 14. (Left) Radiograph showing the presence of a C-shaped root on the mandibular left second molar. (Right) Radiograph of the mandibular right second molar showing two separate roots. Case is a 34 year old female.

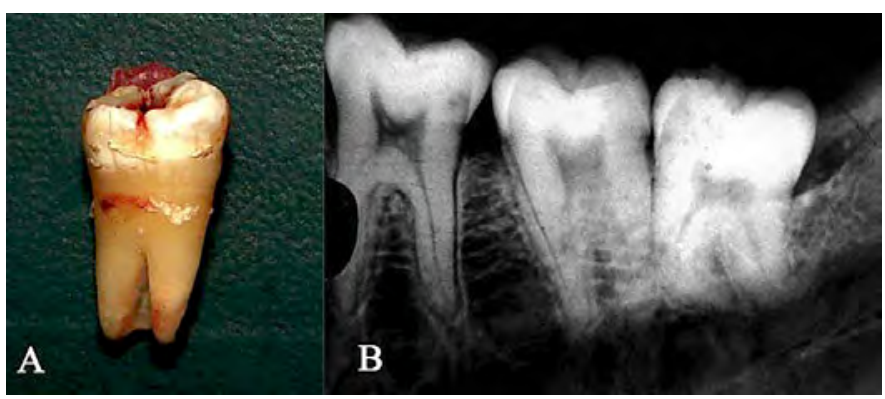

Fig. 15. (Left) Extracted mandibular left second molar having a C-shaped root. (Right) Radiograph of the mandibular right second molar having two separate roots. Case is a 37 year old male.

to have the tooth extracted. It was fond that this tooth had a C-shaped configuration (Fig. 15A). According to Fan et al. (2004), the root was of Type II. A contralateral periapical radiograph (Fig. 15B.) showed that the antimere had two separate roots. Presence of a C-shaped root was diagnosed from the radiograph according to the criteria of Fan et al. (2004) of having a fused root, presence of a longitudinal groove on the root, and our assessment of the coronal, middle and apical third of root canal indicated the presence of $\mathrm{C}$-shaped root.

\section{Mandibular Second Molars}

\section{Presence of an extra root (Radix entomolaris and Radix paramolaris)}

\section{Report 16}

A 23 year old female was referred for root canal treatment on the mandibular right second molar. The tooth was diagnosed with irreversible pulpitis, and root canal treatment was undertaken. Based on the diagnostic periapical radiograph that this tooth had an extra root, a radix entomolaris (Fig. 16A), which was confirmed during root canal treatment (Fig. 16B). A contralateral radiograph showed that that tooth had two separate roots (Fig. 16C).

\section{Report 17}

A 45 year old male reported with the complaints of pain in mandibular left second molar, and he was diagnosed with irreversible pulpitis and root canal treatment was undertaken. The periapical radiograph showed that this molar had an extra root radix entomolaris (Fig. 17A) and this was confirmed during root canal treatment (Fig. 17B). The radiograph of the contralateral tooth showed that it had a fused root (Fig. 17C). Based on the classification by Ribeiro and Consolaro (1997), cases 16, 17 had type I curvature. 

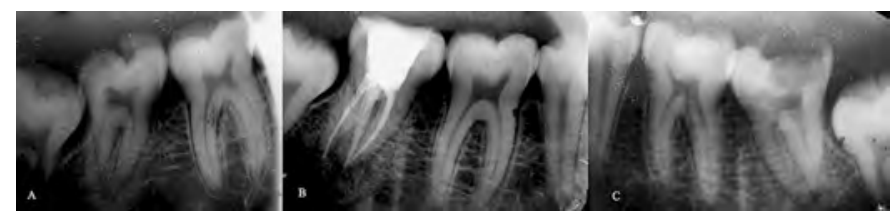

Fig. 16. (Left) Preoperative diagnostic radiograph showing presence of a radix entomolaris on themandibular right second molar. (Center) Post-obturation radiograph confirming the presence of a radix entomolaris on the mandibular right second molar. (Right) Radiograph of mandibular left second molar showing only two roots. Case is a 23 year old female.

\section{Mandibular Third Molars}

\section{Report 18}

A 46 year old male was referred for root canal treatment of the mandibular right third molar, but because of inadequate mouth opening after consulting with the referring doctor it was decided to extract the tooth. Inspection showed that the molar had three roots with an extra root, a radix paramolaris (Fig. 18A). The contralateral tooth (Fig. 18B) had only two separate roots.

\section{Report 19}

A 54 year old male reported with a complaint of pain in the mandibular left third molar. On examination the subject was diagnosed with irreversible pulpitis, and root canal treatment was recommended. But the patient was unwilling, and this third molar was extracted. This third molar had an extra root, a radix entomolaris (Fig. 19A). The contralateral radiograph disclosed that the tooth had two separate roots (Fig. 19B).

\section{Report 20}

A 21 year old male was referred with a complaint of pain in the mandibular left third molar. On examination, a diagnosis of irreversible pulpitis due to carious pulp exposure was made, and root canal treatment was recommended. From the panoramic radiograph (Fig. 20) the mandibular right third molar had an extra root

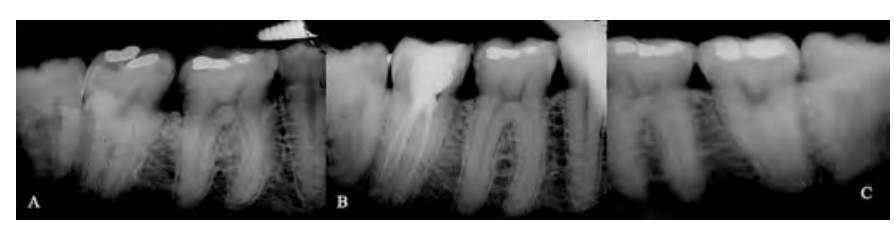

Fig. 17. (Left) Preoperative diagnostic radiograph showing presence of acradix entomolaris on the mandibular left second molar. (Center) Post-obturation radiograph confirming the presence of a radix entomolaris on the mandibular left second molar. (Right) Radiograph of the mandibular right second molar showing fused roots. Case is a 45 year old male.

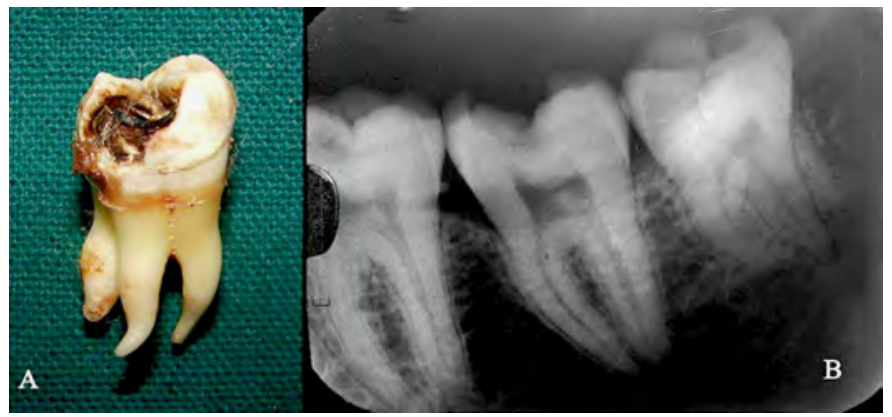

Fig. 18. (Left) An extracted mandibular right third molar with a radix paramolaris. (Right) Radiograph of the mandibular left third molar showing only presence of two roots.

radix paramolaris. The contralateral tooth had only two separate roots. Based on Ribeiro and Consolaro's (1997) classification of radix entomolaris curvature, cases 18, 20 had type III curve and case 19 had type I curve.

\section{Maxillary Second Molars}

\section{Report 21}

A 22 year old female was referred with a complaint of pain in both maxillary right and left second molars. On examination, a diagnosis of irreversible pulpitis with carious pulp exposure was made for both teeth. It was noted from the periapical radiograph of the maxillary left tooth (Fig. 21A) that its palatal root outline was blurred and an extra palatal root canal was suspected. An extra palatal root canal in the maxillary left second molar was confirmed during treatment (Fig. 21B). The contra lateral radiograph revealed that that tooth had only one palatal root canal (Fig. 21C). Based on Vertucci 's (1984) root canal classification, it was a Type IV canal configuration.

\section{DISCUSSION}

The variability of root canal morphology and root numbers represents a challenge to both endodontic

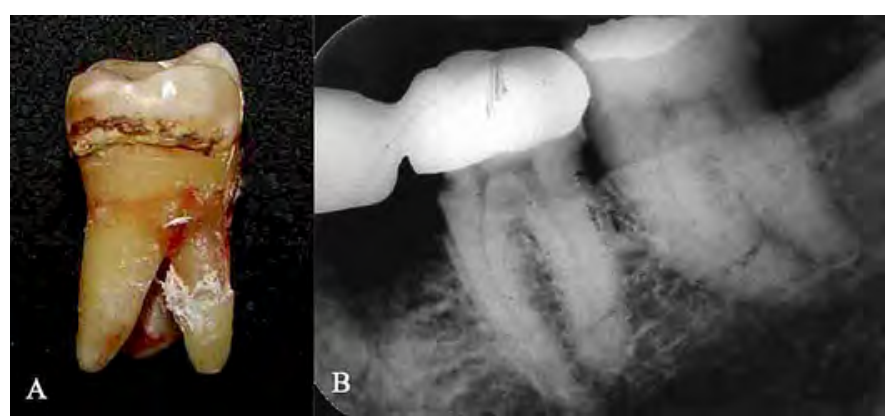

Fig. 19. (Left) An extracted mandibular left third molar with a radix entomolaris. (Right) Radiograph of the mandibular right third molar showing only presence of two roots. Case is a 54 year old male. 


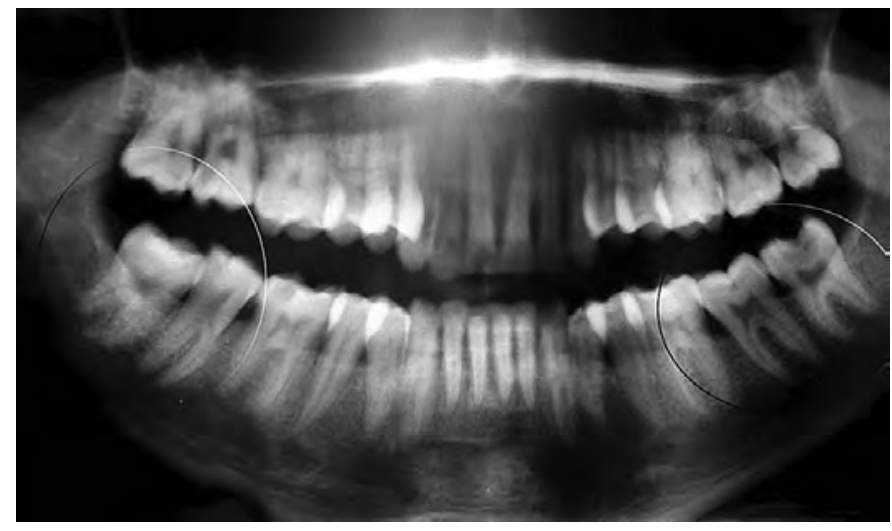

Fig. 20. A panoramic radiograph showing mandibular right third molar having extra root radix paramolaris and a mandibular left third molar showing two separate roots. Case is a 21 year old male.

diagnosis and treatment. Besides the root canal variability, the fluctuating asymmetry presented in these cases reiterates the need for the observer to have a greater understanding of root morphology. Fluctuating asymmetry in root form has been reported and studied exclusively in very limited ways. Bilateral asymmetry has been reported for each group of teeth from different populations, rather than reporting from one group and studying bilateral asymmetry in that population for various tooth types. This is the first time that a case report is presented with fluctuating asymmetry in root aberrations being highlighted.

Review of literature by Cleghorn et al. (2007) reports that incidence of mandibular second premolars with two separate roots is very low and it has been put at an average of $0.4 \%$. In a study by Iyer et al. (2006) among a South Indian group it was concluded that mandibular second premolars with two roots had an incidence of $6.2 \%$, but the study failed to mention how the radiographs were interpreted for root variations. Also, in the same study, bilateral occurrence of root variation was put at $3.2 \%$ but there is no mention which type of root aberrations

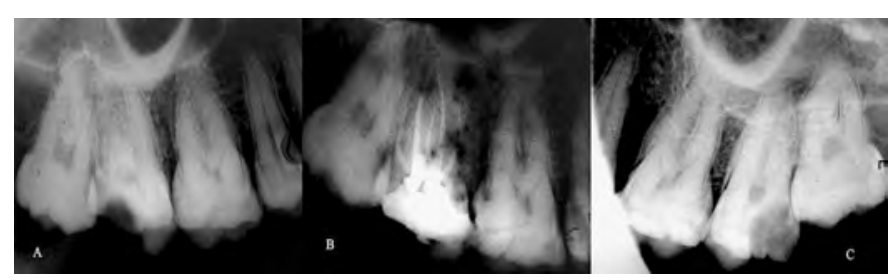

Fig. 21. (Left) Pre-operative diagnostic radiograph suggesting the presence of extra palatal root canal on the maxillary left second molar. (Center) Post-obturation radiograph confirming the presence of the extra palatal root canal on the maxillary left second molar. (Right) Radiograph of the maxillary right second molar showing only one palatal root canal. Case is a 22 year old female. were encountered or for on which mandibular premolar was affected. This bilateral incidence of $3.2 \%$ is much lower than reported by Sabala et al. (1994) for bifurcated mandibular second premolar root, which he reported it at $54.5 \%$ in a Caucasian population.

In contrast to the study by Iyer et al. (2006), the extrarooted mandibular second premolars in the present study occurred only unilaterally. In agreement with Iyer et al. (2006) where it was reported that after Vertucci Type I, the Type IV canal had the highest incidence, in the present cases all the mandibular second premolars with an extra root had Vertucci Type IV canals.

The incidence of three-rooted mandibular first molars in an Indian group has been reported at 5.97\%, and bilateral occurrence of this aberration only at $37.14 \%$ (Garg et al. 2010). In agreement with the present study, cases presented here only occurred unilaterally.

The C-shaped mandibular root configuration in an Indian group has been reported at $7.5 \%$ of all extracted teeth examined; bilateral incidence of this root aberration has not been mentioned in that study (Neelakantan et al. 2010). Sabala et al. (1994) reported that, in a Caucasian group, the bilateral occurrence of a C-shaped root in mandibular second molars is $72.7 \%$. In contrast to this study, we found four cases of mandibular second molars with a C-shaped root occurring only unilaterally.

A mandibular second molar with three roots has been reported for an Indian group at $8.98 \%$ of all the extracted teeth examined, and bilateral occurrence of this root aberration has not been mentioned (Neelakantan et al. 2010). In the Neelakantan study, presence of an extra root was in the mesiolingual position; however, in the case presented here, the extra root was in the distolingual position (radix entomolaris). This extrarooted mandibular second molar occurred only unilaterally.

A mandibular third molar with three roots has been reported with an incidence of about $5 \%$ in Turkish and Caucasian populations; bilateral occurrence of this aberration has not been mentioned (Sidow et al. 2000; Sert et al. 2010). The cases presented here occurred only unilaterally, and two cases had the extra root present lingually (radix entomolaris), and one case had the extra root present buccally (radix paramolaris). A study of variation in mandibular third molar root morphology is lacking for the Indian population.

Maxillary second molars with two palatal canals have a low incidence at $1.4 \%$ for a Caucasian group, and bilateral occurrence of this aberration has not been reported (Peikoff et al. 1996). The one case reported here with two palatal canals occurred only unilaterally. Maxillary second molar root canal variation for Indian populations is lacking.

Very few cases of root aberrations record the bilateral occurrence of root number (De Moor 2002). To our knowledge, the only study that exclusively 
recorded bilateral occurrence of root variations from a Caucasian population is the study by Sabala et al. (1994), and this study concluded that root aberrations occur bilaterally approximately $60 \%$ of the time. This is in contradiction to the present case reports where all of the root aberrations were present unilaterally. This variation in unilateral occurrence of the root aberration may be due to differences in the populations reported.

Ethnic differences in root phenotypes are an established phenomenon (Scott and Turner 2008). Root number and morphological studies for indigenous subgroups in India are very limited. Except for one study about the incidence of three-rooted mandibular first permanent molars, others do not mention the bilateral occurrence of root aberrations or fluctuating asymmetry (Garg et al. 2010). Human diversity in India is defined by 4,693 different, documented population groups that include 2,205 major communities, 589 segments and, 1,900 territorial units spread across the country (Singh 1988). Structuring of the subgroups has been reported in a genetic study (Kashyap et al. 2006). Since tooth and root development is strongly controlled by genetic factors, a more detailed study of root variations in different subgroups in India is warranted. A detailed study about root variations in maxillary and mandibular premolars and molars and their bilateral occurrence in a Tamil speaking population in Madurai, South India; is being undertaken by the authors through the Department of Anthropology, University of Madras.

\section{CONCLUSIONS}

1. From the case reports presented here it is seen that for the Tamil speaking population group in Madurai, South India, fluctuating asymmetry is occurring for various types of root aberrations in permanent premolars and molars.

2. Fluctuating asymmetry in root aberrations will be of importance to clinical dentists, dental anthropologists and dental morphologists.

3. A more detailed study on the incidence of root aberrations in permanent premolars and molars of local population group is necessary with greater emphasis on occurrence of fluctuating asymmetry for root aberrations.

\section{REFERENCES CITED}

Baume RM, Crawford MH (1979) Discrete dental asymmetry in Mexico and Belize. J Dent Res 58:1811.

Carlsen O, Alexandersen V (1990) Radix entomolaris identification and morphology. Scand J Dent Res 98:363-373.

Carlsen O, Alexandersen V (1991) Radix paramolaris in permanent mandibular molars: identification and morphology. Scand J Dent Res 99:189-195.

Cleghorn BM, Christie WH, Dong CC (2007) The root and root canal morphology of the human mandibular second premolar: a literature review. J Endodont 33:1031-1037.

De Moor RJG (2002) C-shaped root canal configuration in maxillary molars. Int Endodont J 35:200-208.

Dibennardo R, Bailit HL (1978) Stress and dental asymmetry in a population of Japanese children. Am J Phys Anthropol 48:89-94.

Fan B, Cheung GSP, Fan M, Gutmann JL, Fan W (2004) C-shaped canal system in mandibular second molars: Part II-radiographic features. J Endodont 30:904-908.

Garg AK, Tewari RK, Kumar A, Hashmi SH, Agarwal N, Mishra SK (2010) Prevalence of three rooted mandibular permanent first molar among the Indian population. J Endodont 36:1302-1306.

Garn SM, Lewis AB, Kerewsky RS (1966) Bilateral asymmetry and concordance in cusp number and crown morphology of the mandibular first molar. J Dent Res 45:1820.

Iyer $\mathrm{VH}$, Indira $\mathrm{R}$, Ramachandran $\mathrm{S}$, Srinivasan MR (2006) Anatomic variations in mandibular premolars in Chennai population. Indian J Dent Res 17:7-10.

Kashyap VK, Guha S, Sitalaxmi T, Bindu GH, Hasnain SE, Trivedi R (2006) Genetic structure of Indian populations based on fifteen autosomal microsatellite loci. BMC Genetics 7:28-36.

Kieser JA. Groeneveld HT, Preston CB (1986) Fluctuating dental asymmetry as a measure of odontogenic canalization in man. Am J Phys Anthropol 71:437444.

Kieser JA, Groeneveld HT (1988) Fluctuating odontometric asymmetry in an urban South African black population. J Dent Res 67:1200.

Lundström A (1961) Some asymmetries of the dental arches, jaws and skull, and their etiological significance. Am J Orthod 47:81-106.

Lundsröm A (1963) Tooth morphology as a basis for distinguishing monozygotic and dizygotic twins. Am J Hum Genet 15:34-43.

Neelakantan P, Subbarao C, Subbarao CV, Ravindranath M (2010) Root and canal morphology of mandibular second molars in an Indian population. J Endodont 36:1319-1322.

Noss JF, Scott GR, Potter RH, Dahlberg AA (1983) Fluctuating asymmetry in molar dimensions and discrete morphological traits in Pima Indians. Am J Phys Anthropol 61:437-445.

Peikoff MD, Christie WH, Fogel HM (1996) The maxillary second molar: variations in the root number of roots and root canals. Int Endodont J 29:365-369.

Ribeiro FC, Consolaro (1997) Importancia clinica antropologica de la raiz distolingual en los molars inferiores permamented. Endodoncia 15:72-78.

Sabala CL, Beneati FW, Neas BR (1994) Bilateral root or root canal aberrations in a dental school patient 
population. J Endodont 20:38-42.

Schafer E, Breuer D. Janzen S (2009) The prevalence of three-rooted mandibular permanent first molars in a German population. J Endodontics 35202-205.

Scott GR, Turner CG II (2000). Biological considerations: ontogeny, asymmetry, sex dimorphism, and intertrait association. In: Scott GR, Turner CG II, editors. The anthropology of modern human teeth. Dental morphology and its variation in recent human populations. Bristol, UK: Cambridge University Press, p 96-105.

Scott GR, Turner CG II (2008) History of dental anthropology. In: Irish JD, Nelson GC, editors. Technique and Application in Dental Anthropology. New York: Cambridge University Press, p 10-35.

Sert S, Sahinkesen G, Topcu FT, Eroglu SE, Oktay EA (In press) Root canal configurations of third molar teeth. A comparison with first and second molars in the Turkish population. Aust Endodont J.

Sidow SJ, West LA, Liewehr R, Loushine RJ (2000) Root canal morphology of human maxillary and mandibular third molars. J Endodont 26:675-678.
Singh KS (1988) India's Communities. People of India. National series volume IV. India. New Delhi: Oxford University Press.

Townsend GC (1981) Fluctuating asymmetry in deciduous dentition of Australian Aboriginals. J Dent Res 60:1849.

Tu MG, Tsai CC, Jou MJ, Chen WL, Chang YF, Chen SY, Cheng HW. (2007). Prevalence of three-rooted mandibular molars among Taiwanese individuals. J Endodont 33:1163-1166.

Van Valen L (1962) A study of fluctuating asymmetry. Evolution 16:125-142.

Vertucci FJ (1984) Root canal anatomy of the human permanent teeth. Oral Surg Oral Med Oral Path Oral Rad Endodont 58:589-599.

Walker RT, Quackenbush LE (1985) Three rooted lower first permanent molars in Hong Kong Chinese. Br Dent J 159:298-299.

Wetherell J, Winning T, Townsend GC (2004) Localized asymmetry in human dental crown form-an interesting case. Dental Anthropology 17:18-23.

\section{DAA Subscription}

The secretary-treasurer of the Dental Anthropology Association is Dr. Loren R. Lease of Youngstown State University.

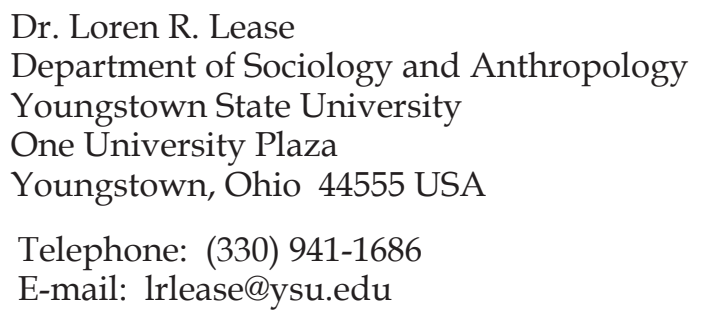

Dr. Loren R. Lease

Department of Sociology and Anthropology

Youngstown State University

One University Plaza

Youngstown, Ohio 44555 USA

Telephone: (330) 941-1686

E-mail: lrlease@ysu.edu

Dental Anthropology now is published electronically and e-mailed to all members as a PDF. If you also want to receive a hard copy, be sure to make this clear on the membership form at the DAA website or contact Loren.

Speed communication about your membership by contacting Loren directly (other officers may not have current membership information). 